\title{
Short Term Electric Load Forecasting based on Artificial Neural Networks for Weekends of Baghdad Power Grid
}

\author{
Ibraheem K. Ibraheem, Ph.D \\ Department of Electrical Engineering \\ College of Engineering, Baghdad University
}

\author{
Mohammed Omar Ali \\ Department of Electrical Engineering \\ College of Engineering ,Baghdad University
}

\begin{abstract}
This work presents proposed methodsfor short term power load forecasting (STPLF) for the governorate of Baghdad using two different models of Artificial Neural Networks (ANNs). The two models used in this work are the multi-layer perceptron (MLP) model trained with Levenberg-Marquardt Back Propagation (BP) algorithm and Radial Basis Function (RBF) neural network. Inputs to the ANN are thepast loadsvalues and the output of the ANN is the load forecast for the weekends of certain months for Baghdad governorate. The data is divided into two parts where half of them was used for training and the other half was used for testing the ANN. Simulations were achieved by MATLAB software with the aid of Neural networks toolbox, where the data obtained for the Iraqi national grid were rearranged and preprocessed. Finally, the simulations results showed that the forecasted load values for the Baghdad governorate by the proposed methods were very close to actual ones as compared with the traditional methods.
\end{abstract}

\section{General Terms}

Load demand, neural networks, load prediction, artificial intelligence, energy consumption.

\section{Keywords}

Load forecasting, multilayer perceptron, radial basis neural networks (RBF), Back Propagation.

\section{INTRODUCTION}

Power load forecasting (PLF) accurately plays a very important role for electric utilities in a competitive environment created by the electric industry deregulation. An electric company is confronted with many economical and technical problems in operation, planning and control of an electric energy system since customers require high quality electric energy to be supplied in a secure and economic manner [1]. PLF helps an electric utility by making important decisions on generating, interchanging, and purchasing electric power, load switching, and infrastructure development. Besides PLF is crucial for energy suppliers, financial institutions, and others involved in electric energy generations, transmission, distribution, and markets [2]. Moreover, PLF is playing a key role in reducing the generation cost, it is also essential to the reliability of power systems. The system operators use the load forecasting result as a basis of off-line network analysis to determine if the system might be vulnerable. If so, corrective actions should be prepared, such as load shedding, power purchases and bringing peaking units on line [3]. According to forecasting time period, PLF can be divided into three categories [4]: Short Term Power Load Forecast (STPLF) which is usually from one hour to one week, and it is primarily used for the day-to-day operation, control and scheduling of the power systems. Medium Term Power Load Forecast (MTPLF) which is usually from a week to a year and it is generally used for the maintenance and scheduling programs of fuel supplies. Finally, Long Term Power Load Forecast (LTPLF) which is longer than a year and it is primarily used for power system development planning. Many researchers have investigated power load forecasting;in [5] the authors presented a multilayer feed-forward neural network model with the aim to compare the forecasting accuracy of a time-series and an ANN-based model. While researchers in [6] used a three layer feed-forward neural network and a back-propagation training algorithm, so that electricity prices could be considered as one of the main factors affecting the load in deregulated markets. A supervised neural network-based model has been proposed by [7] to forecast the load in the Nigerian power system. [8] Proposed a study of design a neural network model called Elman recurrent network by using MATLAB software to simulate the power load forecasting. The research presented by [9] suggested Models based on the so-called Multi- Layer Perceptron (MLP) network to solve the problem of short term load forecasting. Finally, [10] presented a new method for STPLF to predict the demand in the future. The main objective of this study was to analyze the profile or pattern of the forecasted load and to predict the load demand during weekends. [11] Proposed a multi-parameter regressionmethod for forecasting which has error within tolerable range.Particle swarm optimization has been applied on STPLF in [12], while [13] used a new approach for short-term load forecasting (STLF), where curve fitting prediction and time series models are used for hourly loads forecasting of the week days combined with genetic algorithm.

\section{ARTIFICIAL NEURAL NETWORKS}

Artificial Neural Networks (ANNs) are a data processing system consisting of a large number of simple, highly interconnected processing elements inspired by the biological system and designed to simulate neurological processing ability of human brain [14]. A generic artificial neural network can be defined as a computational system consisting of a set of highly interconnected processing elements, called neurons, which process information as a response to external stimuli. An artificial neuron is a simplistic representation that emulates the signal integration and threshold firing behavior of biological neurons by means of mathematical equations [15]. An artificial neuron and its model is shown in Figure 1.

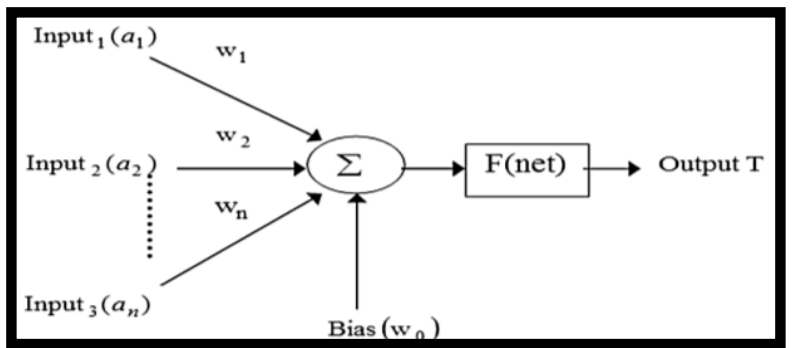

Fig. 1: The basic model of an artificial neuron. 


\section{PROPOSED MLP AND RBF ANN MODELS FOR STPLF}

In this section, models of MLP and RBF are presented to forecast the output load of weekends for certain months in 2012 for Baghdad City asa case study. The proposed structure for predicting demands consisted of four inputs for training and one output.

\subsection{Designing MLP and RBF ANN models for STPLF}

MLP is a popular architecture of ANN andcan be used for STPLF. In this work, MLP has been trained with Levenberg Marquardt BP algorithm and the transfer function within the network was the sigmoid nonlinear activation function with only one neuron in its output layer as shown in figure 2 . This neuron gave the output value, which contains the predicted value of the weekend load.

The second type of ANN model used in this work to solve the problem of STPLF was RBF neural network model. RBF model consists of three layers. The input layer has neurons with a linear function and the hidden neurons are processing units that perform the RBF function. The output neuron is a summing unit to produce the output as a weighted sum of the hidden layer outputs, figure 3 depicts the proposed model of the FBF NN structure.

Selecting the basis function is not crucial to the performance of the network; the most common is the Gaussian basis function, whichis used in this study. Designing ANN models follow a number of systemic procedures. In general, there are five basic steps:- for Baghdad power grid have been collected from the Iraqi Operation and Control Office for 3 years from 2010 to 2012 .

2. Data Preprocessing: After data collection, two preprocessing procedures are conducted to train the ANNs more efficiently. These procedures are: (a) solve the problem of missing data and (b) normalize data

3. Building the Network: At this stage, the number of hidden layers, neurons in each layer, transfer function in each layer, training function and performance function were specified.

4. Training the Network: During the training process, the weights are adjusted in order to make actual outputs (forecasted) close to the target (measured) outputs of the network. MATLAB provided built-in transfer functions linear (purelin), hyperbolic tangent sigmoid (logsig) and Logistic (tansig) which were used in this work.

5. Testing the Network: The next step was to test the performance of the developed model. In order to evaluate the performance of ANN models such as the mean square error (MSE), MSE provided information on the STPLF performance, which is a measure of the variation of predicted values around the measured data. The lower the MSE was, the more accurate was the estimation. It can be calculated from Eq. (1) below:

$$
M S E=\frac{1}{N} \sum_{i=1}^{N}\left[L_{a}(n)-L_{p}(n)\right]^{2}(1)
$$

Where $L_{a}(n)$ is the actual load, $L_{p}(n)$ is the forecasted or predicted value of load, and $N$ is the number of data points.

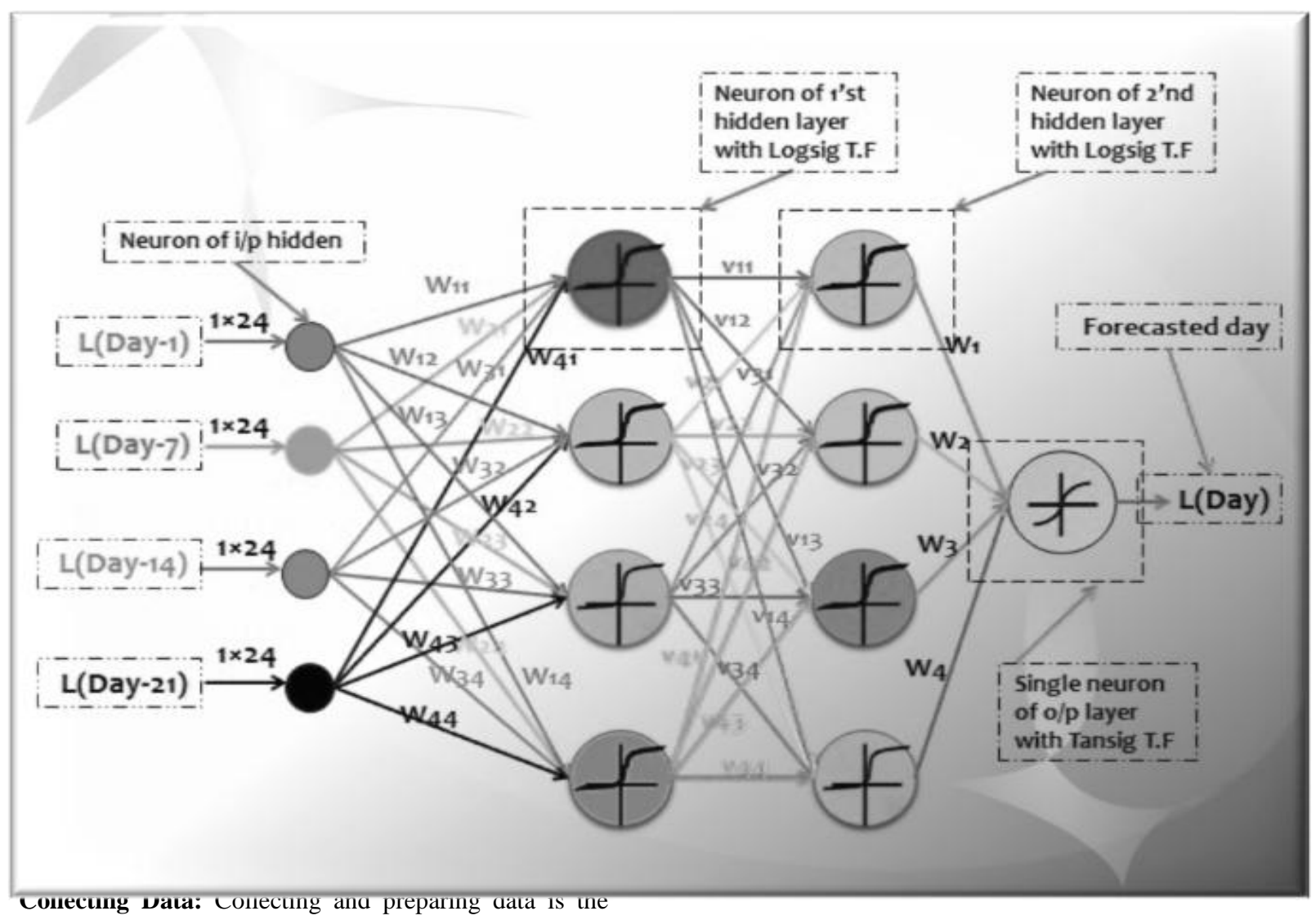

first step in designing ANN models. In this work, all data

Fig. 2: The proposed structure of MLP Neural network model to forecast the weekend load. 


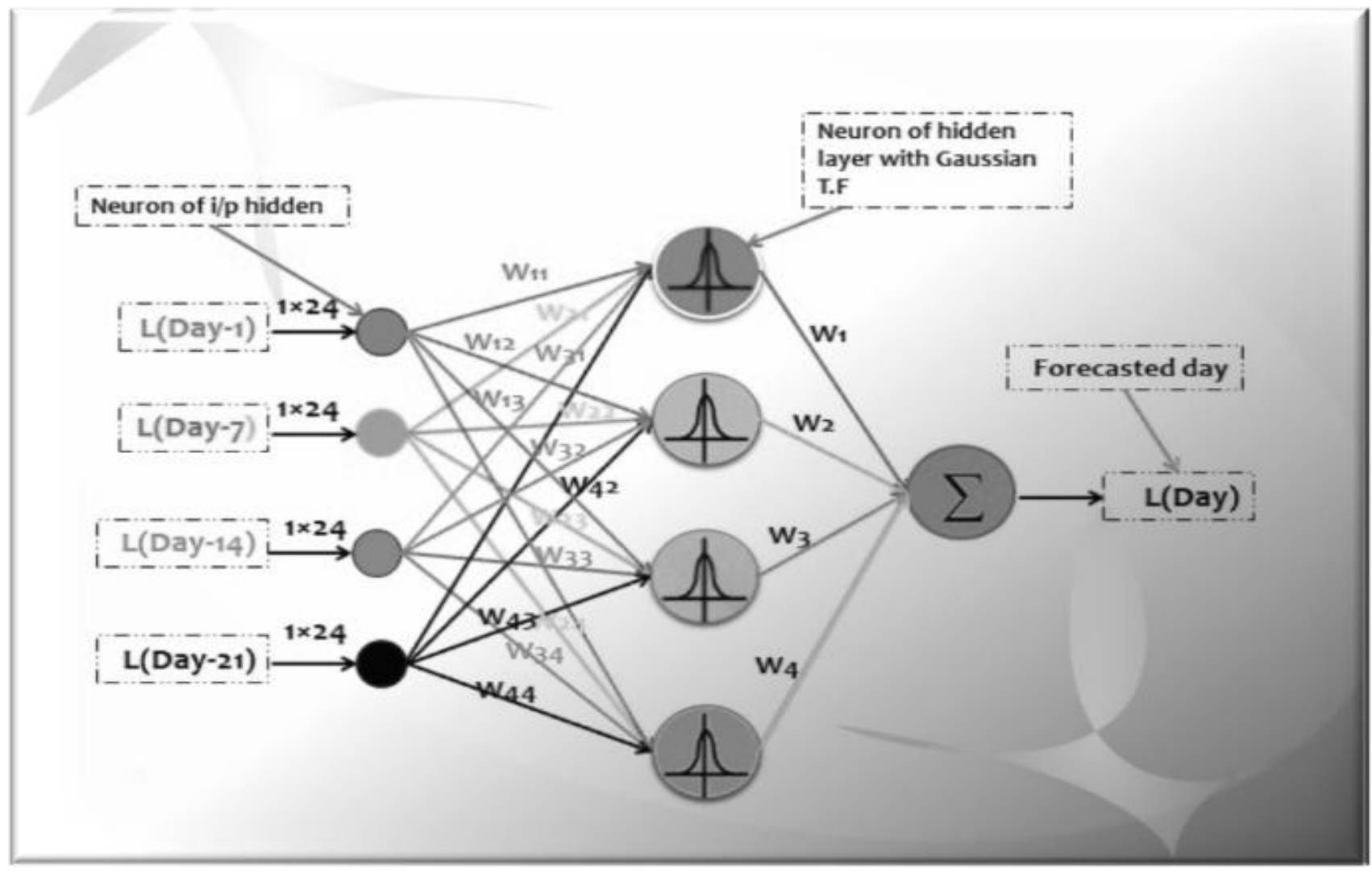

Fig. 3: The proposed structure of RBF model to forecast a weekend load.

\subsection{Learning Mechanism of MLP and RBF ANN models for STPLF}

The learning mechanism of MLP and RBF ANN models for STPLF can be summarized in the following steps:-

1. The program started by reading data from an excel file. The function of "xlsread" was used to read the data specified in the excel file.

2. The loaded data was normalized to remain within the range $(0-1)$. The actual load was scaled using the expression represented in Eq. (2):-

$$
L_{s}=\frac{L}{L_{\max }}(2)
$$

Where $L_{S}$ is the scaled or normalized load, $L$ is the actual load in $\mathrm{MW}$, and $L_{\max }$ is the maximum load during the day in MW.

3. The neural network was constructed by using the function (Newff) for MLP or by using the function (Newrb) for RBF; each of them has one input layer, one hidden layer and one output layer. The transfer functions for hidden and output layers were "tansig" and "logsig", respectively. The number of neurons in input and output layers was closely related with the sample, according to the historical data, but the number of neurons in hidden layer could be taken from the empirical formula :

$$
i=\sqrt{(n+m)}+a(3)
$$

Where $i$ is the number of neurons in hidden layer, $n$ is the number of neurons in input layer, $m$ is the number of neurons in output layer, and $a$ is a constant and $1<\mathrm{a}<10$.

4. The network is next configured as follows:

net.trainFcn = LM;

net.trainparam.min_grad $=0.00000001$;

net.trainParam.epochs $=1000$

net.trainParam.lr $=0.25$;

net.trainParam. $\mathrm{mc}=0.8$;

net.trainParam.max_fail $=50$;

Where trainFcn" defines the function used to train the network. It can be set to the name of any Training function (LM ='trainlm'; Levenberg-Marquardt $\quad$ BP), "trainparam.min_grad" denotes the minimum performance gradient, "trainParam.epochs" denotes the maximum number of epochs to train, "train Param.lr" denotes the learning rate, "trainParam.mc"denotes the Momentum term,"trainParam.max_fail" denotes the maximum validation failures.

5. The network was trained with previous data by using supervised Levenberg -Marquardt BP algorithm. In this study, the data was from (2010) to (2011) for training.

6. The network was simulated or tested after completing its training. This process is achieved by calling the function "sim". 
7. The output from the constructed network was denormalized in order to compare it with the measured data and then the results were written in excel file.

8. The last step was performed by calling the performance function to calculate and store the performance error statistics like MSE. Fig. 4 shows the programming of MLP and RBF models using MATLAB.
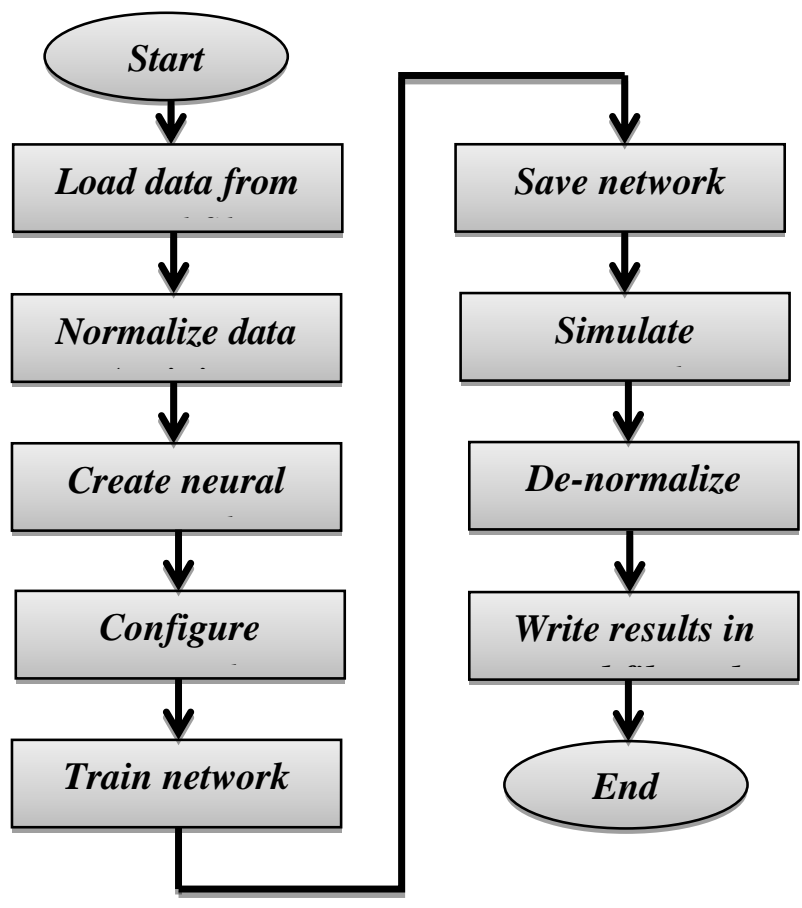

Fig. 4: Flow chart for programming MLP and RBF Models using MATLAB.

\section{SIMULATION RESUTS}

In this section, the simulations on the proposed models of MLP and RBF are presented to forecast the output load of the weekends for certain months in 2012 for Baghdad governorate as a case study. The proposed structure for predicting demands consisted of four inputs for training and one output. Figure 5 shows the relationship between loads in (MW) and time in (hrs) for Sat. 28/Jan./2012 for Baghdad city. As can been seen from the figure that the predicted load values using RBF is closer to the actual loads than that of the MLP. Tables1 and 2 show the training and testing data for forecasting a weekend of Saturday in July 2012 for Baghdad city respectively. Whereas Table 3 presents the actual and predicted load values using MLP and RBF models. Figure 6 shows that the predicted loads using RBF and MLP models to forecast the load values for the weekendfor Fri 27 / July / 2012, while figure 7 is for sat 27 / Oct. / 2012. The figures show that the RBF achieves better performance than MLP over the entire weekend time. It can be seen form the figures that for certain weekend the forecasted load curves lie above the actual load curve (figure 5) while for other weekend it lie below the actual loads (figure 6 ). Figures 6 and 7 shows that the forecasted load values using RBF model exactly fits the actual load curve which means that RBF perform very well than MLP, still the predicted load values using MLP are good as compared with the actual load curve.which means that the results obtained using both models can be trusted and can be used for a practical power system.

Table 1. The training data for forecasting a weekend of Saturday in July 2012 for Baghdad Governorate

\begin{tabular}{|c|c|c|c|c|c|}
\hline & \multicolumn{4}{|c|}{$\begin{array}{l}\text { Input data for training } \\
\text { (MW) }\end{array}$} & \multirow{2}{*}{$\begin{array}{c}\text { Target for } \\
\text { training }\end{array}$} \\
\hline 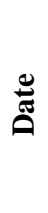 & 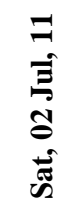 & 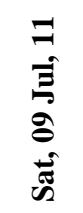 & 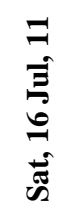 & $\begin{array}{l}\Xi \\
\vec{\Xi} \\
\tilde{\Xi} \\
\vec{\Xi} \\
\vec{E}\end{array}$ & \\
\hline 1 & 2979 & 3142 & 3140 & 3112 & 3205 \\
\hline 2 & 2882 & 3067 & 3065 & 3037 & 3127 \\
\hline 3 & 2954 & 3142 & 3140 & 3112 & 3205 \\
\hline 4 & 2990 & 3180 & 3178 & 3150 & 3243 \\
\hline 5 & 2954 & 3142 & 3140 & 3112 & 3205 \\
\hline 6 & 3080 & 3275 & 3273 & 3244 & 3340 \\
\hline 7 & 3224 & 3408 & 3405 & 3375 & 3475 \\
\hline 8 & 3206 & 3408 & 3405 & 3375 & 3475 \\
\hline 9 & 3242 & 3426 & 3424 & 3394 & 3494 \\
\hline 10 & 3278 & 3464 & 3462 & 3431 & 3533 \\
\hline 11 & 3296 & 3483 & 3481 & 3450 & 3552 \\
\hline 12 & 3343 & 3517 & 3515 & 3484 & 3587 \\
\hline 13 & 3347 & 3521 & 3519 & 3487 & 3591 \\
\hline 14 & 3347 & 3521 & 3519 & 3487 & 3591 \\
\hline 15 & 3386 & 3597 & 3594 & 3562 & 3668 \\
\hline 16 & 3314 & 3521 & 3519 & 3487 & 3591 \\
\hline 17 & 3350 & 3559 & 3556 & 3525 & 3629 \\
\hline 18 & 3386 & 3597 & 3594 & 3562 & 3668 \\
\hline 19 & 3422 & 3635 & 3632 & 3600 & 3707 \\
\hline 20 & 3368 & 3673 & 3670 & 3637 & 3745 \\
\hline 21 & 3278 & 3559 & 3556 & 3460 & 3629 \\
\hline 22 & 3170 & 3445 & 3399 & 3412 & 3514 \\
\hline 23 & 3098 & 3290 & 3261 & 3259 & 3303 \\
\hline 24 & 3051 & 3207 & 3205 & 3177 & 3271 \\
\hline
\end{tabular}


Table 2. The testing data for forecasting a weekend of Saturday in July 2012 for Baghdad Governorate

\begin{tabular}{|c|c|c|c|c|c|}
\hline & \multicolumn{4}{|c|}{$\begin{array}{l}\text { Input data for testing } \\
\text { (MW) }\end{array}$} & \multirow{2}{*}{ 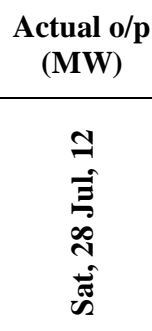 } \\
\hline 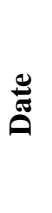 & 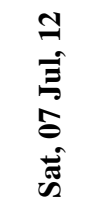 & 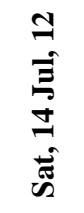 & 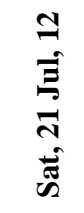 & $\begin{array}{l}\text { I } \\
\bar{E} \\
\vec{\Xi} \\
\vec{\Xi} \\
\vec{E}\end{array}$ & \\
\hline 1 & 2951 & 3260 & 3315 & 3320 & 3227 \\
\hline 2 & 2825 & 2886 & 2937 & 2941 & 2858 \\
\hline 3 & 2744 & 3052 & 3105 & 3110 & 3022 \\
\hline 4 & 2788 & 3177 & 3231 & 3236 & 3145 \\
\hline 5 & 3033 & 3135 & 3189 & 3194 & 3104 \\
\hline 6 & 3399 & 3509 & 3567 & 3573 & 3473 \\
\hline 7 & 3440 & 3592 & 3651 & 3658 & 3555 \\
\hline 8 & 3562 & 3633 & 3693 & 3700 & 3596 \\
\hline 9 & 3603 & 3716 & 3777 & 3784 & 3677 \\
\hline 10 & 3725 & 3799 & 3861 & 3868 & 3759 \\
\hline 11 & 3766 & 3841 & 3861 & 3868 & 3759 \\
\hline 12 & 3827 & 3903 & 3924 & 3932 & 3821 \\
\hline 13 & 3807 & 3882 & 3903 & 3911 & 3800 \\
\hline 14 & 3807 & 3882 & 4029 & 4037 & 3923 \\
\hline 15 & 3807 & 3882 & 4155 & 4164 & 4046 \\
\hline 16 & 3990 & 4069 & 4176 & 4185 & 4066 \\
\hline 17 & 4010 & 4090 & 4155 & 4164 & 4046 \\
\hline 18 & 4010 & 4090 & 4155 & 4164 & 4046 \\
\hline 19 & 4010 & 4090 & 4113 & 4121 & 4005 \\
\hline 20 & 4010 & 4090 & 4071 & 4079 & 3964 \\
\hline 21 & 4002 & 4048 & 4029 & 4037 & 3932 \\
\hline 22 & 3888 & 3965 & 4010 & 4037 & 3923 \\
\hline 23 & 3848 & 3922 & 3960 & 3995 & 3882 \\
\hline 24 & 3609 & 3675 & 3725 & 3742 & 3636 \\
\hline
\end{tabular}

Table 3. Actual output and forecasted load values for RBF and MLP for Sat. 28/July/2012

\begin{tabular}{|c|c|c|c|}
\hline $\begin{array}{l}\text { No. of } \\
\text { hour }\end{array}$ & $\begin{array}{c}\text { Predicted } \\
\text { output using } \\
\text { MLP(MW) }\end{array}$ & $\begin{array}{c}\text { Predicted } \\
\text { output using } \\
\text { RBF(MW) }\end{array}$ & $\begin{array}{l}\text { Actual } \\
\text { output } \\
\text { (MW) }\end{array}$ \\
\hline 1 & 3559.7 & 3388 & 3227 \\
\hline 2 & 3039.3 & 2982.5 & 2858 \\
\hline 3 & 3402 & 3173.9 & 3022 \\
\hline 4 & 3584.3 & 3309.1 & 3145 \\
\hline 5 & 3270.7 & 3241 & 3104 \\
\hline 6 & 3694.4 & 3629.3 & 3473 \\
\hline 7 & 3761 & 3719.4 & 3555 \\
\hline 8 & 3871.3 & 3755.2 & 3596 \\
\hline 9 & 3905.4 & 3844.9 & 3677 \\
\hline 10 & 4048.9 & 3926.8 & 3759 \\
\hline 11 & 4065.2 & 3938.4 & 3759 \\
\hline 12 & 4128 & 4002.3 & 3821 \\
\hline 13 & 4107.7 & 3981 & 3800 \\
\hline 14 & 4149.7 & 4073.6 & 3923 \\
\hline 15 & 4157.8 & 4165.5 & 4046 \\
\hline 16 & 4272.3 & 4231.6 & 4066 \\
\hline 17 & 4285.7 & 4222 & 4046 \\
\hline 18 & 4285.7 & 4222 & 4046 \\
\hline 19 & 4282.9 & 4191.6 & 4005 \\
\hline 20 & 4276 & 4161.1 & 3964 \\
\hline 21 & 4302 & 4116.1 & 3932 \\
\hline 22 & 4165.6 & 4089.5 & 3923 \\
\hline 23 & 4120.1 & 4043.7 & 3882 \\
\hline 24 & 3907 & 3793.9 & 3636 \\
\hline MSE & 4.32E-03 & $1.55 E-03$ & \\
\hline
\end{tabular}




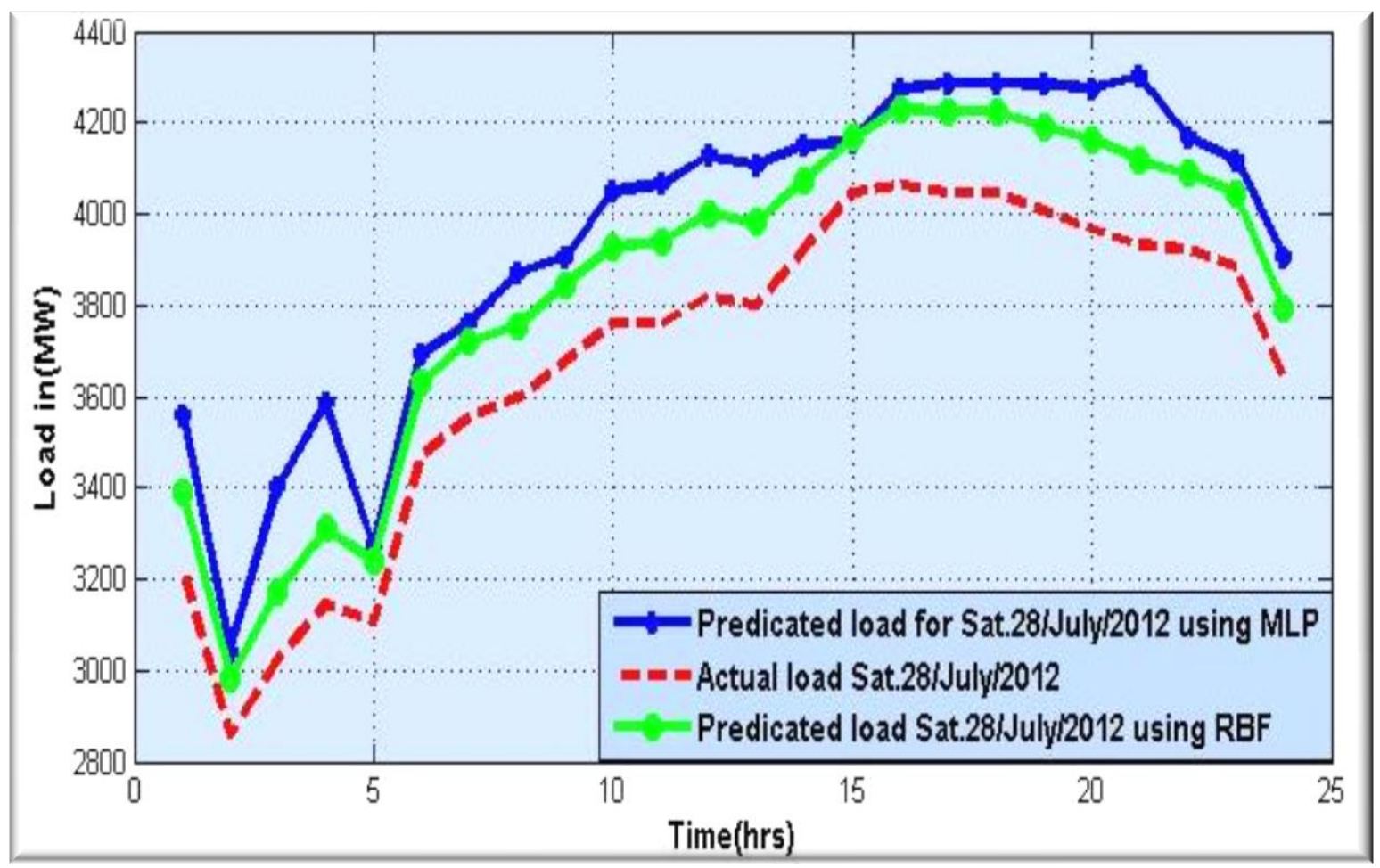

Fig. 5: Actual and predicted load using MLP and RBF for the weekend of Saturday / July / 2012 for Baghdad city.

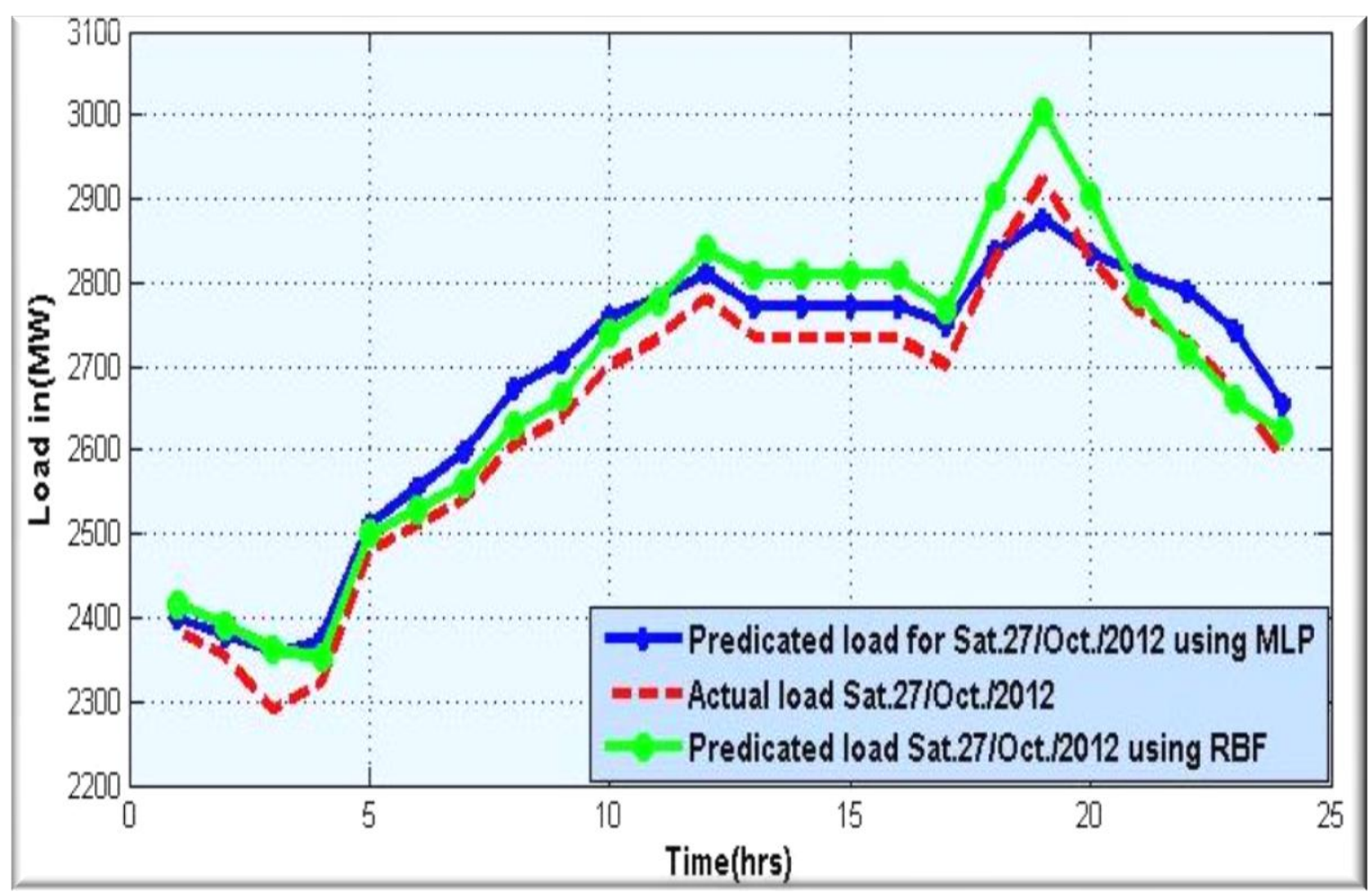

Fig. 6:Actual and predicted load using MLP and RBF of a weekend of Friday / July / 2012 for Baghdad city. 


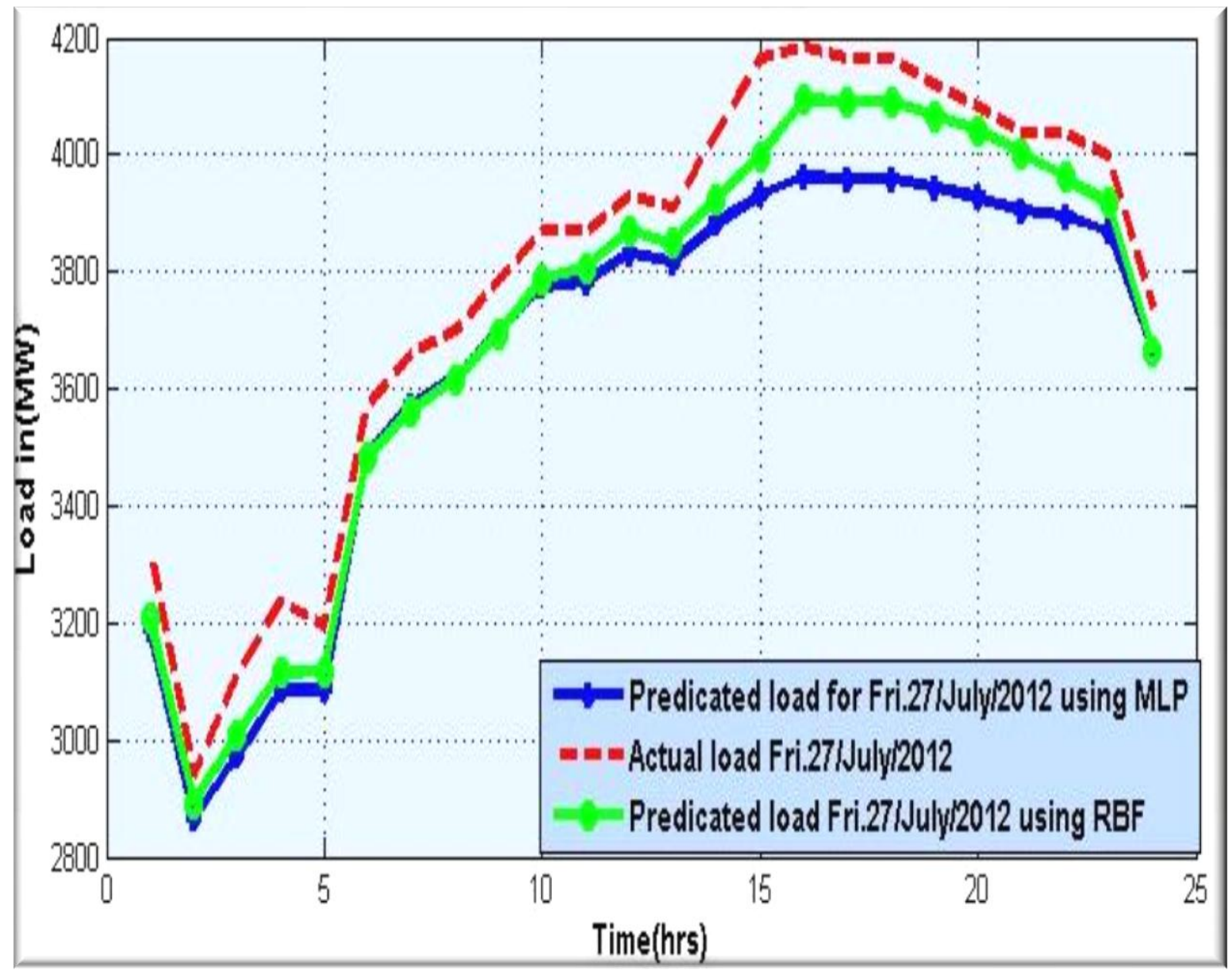

Fig. 7: Actual and predicted load using MLP and RBF of a weekend of Saturday in October 2012 for Baghdad city.

\section{CONCLUSIONS}

In this work, simulations and programming of short-term power load forecasting problem presented for Baghdad city power grid by using two different models of artificial neural networks, the feedforward MLP and radial basis functions RBF models. The two models presented good forecasted load values for the weekends of Baghdad city for certain months, but RBF showed very good performance as compared to MLP. The results obtained showed the effectiveness of the developed method. Based on the results obtained from this work, it can be conclude that ANN models with the developed structure could perform good prediction with least error and finally this neural network could be an important tool for short term power load forecasting.

\section{ACKNOWLEDGMENTS}

Our thanks to the electrical engineering department for providing the necessary software and many thanks to the Iraqi national control center / ministry of electricity for providing us with the data for Iraqi power grid.

\section{REFERENCES}

[1] K. Y. Lee, Y. T. Cha, and J. H. Park.1992. Short-Term Load Forecasting Using An Artificial Neural Network. IEEE Transactions on Power Systems, Vol. 7, No. 1, pp. 124-132.

[2] J. H. Chow.2005. Applied Mathematics for Restructured Electric Power Systems:Optimization, Control, and Computational Intelligence. New York: Springer Verlag, Ch. 12.

[3] G. Gross and F. D. Galiana. 1987. Short-term load forecasting. Proceedings of the IEEE, Vol. 75, No. 12, pp. $1558-1573$.

[4] M.T. Haque and A.M.Kashtiban. 2005. Application of Neural Networks in Power System. A Review, Transactions on Engineering, Computing and Technology Vol. 6, June.

[5] S.S. Sharif and J.H. Taylor. 2000. Short term Load Forecasting by Feed Forward Neural Networks. Proc. IEEE ASME First Internal Energy Conference (IEC), Al Ain, United Arab Emirate.

[6] H. Chen, C. A. Canizares, and A. Singh. 2001. ANNbased Short-term load forecasting in Electricity 
Markets.Proc. IEEE on Power Engineering Society Winter Meeting, Vol. 2, pp. 411-415.

[7] G.A. Adepoju, S.O.A Ogunjuyigbe, and K.O Alawode.2007. Application of Neural Network to Load Forecasting in Nigerian Electrical Power System. The Pacific Journal of Science and Technology, Vol. 8, No. 1 , May.

[8] S. N., A. Yelamali, and K. Byahatti. 2010. Electricity Short term Load Forecasting using Elman Recurrent Neural Network. International Conference on Advances in Recent Technologies in Communication and Computing.

[9] S. K. Sheikh and M. G. Unde. 2012. Short Term Load Forecasting Using ANN Technique. International Journal of Engineering Science and Emerging Technologies, Vol. 1, No. 2, pp.97-107.

[10] N. A. Salim, T.K. Abdul Rahman, M.F. Jamaludin, and M.F. Musa. 2009. Case Study of Short Term Load Forecasting for Weekends. Proc. IEEE Student Conference on Research and Development, Malaysia.

[11] J. P. Rothe, A. K. Wadhwani, and Mrs. S. Wadhwani. 2009. Short Term Load Forecasting Using Multi Parameter Regression. International Journal of Computer Science and Information Security(IJCSIS), Vol. 6, No. 2.

[12] Azzam-ul-Asar, Syed RiazulHassnain, Affan Khan. 2007. Short Term Load Forecasting Using Particle
Swarm Optimization Based ANN Approach,Proceedings of International Joint Conference on Neural Networks, Orlando, Florida, USA, August 12-17.

[13] M. A. Farahat, M. Talaat. 2012. Short-Term Load Forecasting Using Curve Fitting Prediction Optimized by Genetic Algorithms", International Journal of Energy Engineering, 2(2): 23-28.

[14] X. Cui, T. E. Potok and P. Palothingal. 2005. Document Clustering using Particle Swarm Optimization, IEEE, May.

[15] S. Haykin. 1999. Neural Networks", 2nd ed., A Comprehensive Foundation, MacMillan Publishing, Englewood Cliffs, N.J. 